American Journal of Modern Physics
2018; 7(1): $21-30$
http://www.sciencepublishinggroup.com/j/ajmp
doi: $10.11648 / j$. ajmp.20180701.13
ISSN: 2326-8867 (Print); ISSN: 2326-8891 (Online)

\title{
New Trend in Radiation Dosimeters
}

\author{
Aya Mahmoud Hamdy Abaza \\ Radiation Protection Department, Nuclear and Radiological Regulatory Authority (ENRRA), Cairo, Egypt
}

\section{Email address:}

aya_abaza@hotmail.com

\section{To cite this article:}

Aya Mahmoud Hamdy Abaza. New Trend in Radiation Dosimeters. American Journal of Modern Physics. Vol. 7, No. 1, 2018, pp. 21-30. doi: 10.11648/j.ajmp.20180701.13

Received: September 30, 2017; Accepted: October 24, 2017; Published: December 14, 2017

\begin{abstract}
A radiation dosimeter is a device that measures exposure to ionizing radiation. It is used for human radiation protection as a measurement of dose in both medical and industrial processes. This study aimed to identify and highlight the new technology in the types of radiation dosimeters. The study comprises a personal selection of recent reports from radiology journals and Medline searches which highlighted the new trend in radiation dosimeters. Radiation dosimeters and dosimetry systems come in many shapes and forms. They rely on numerous physical effects for storage and readout of the dosimetric signal. The four most commonly used radiation dosimeters are: Ionization chambers; Radiographic films; Thermoluminescent dosimeter systems (TLDs); Silicon diode dosimetry systems (Diodes). There are a variety of electronic dosimetry systems that can monitor any work environment. Electronic dosimeters protect the wearer from the harmful effects of radiation by tracking changes in exposure and keeping an ongoing record of the user's dose over time. Combined with access control systems, it is possible to limit total exposure to radiation and control access to radiological areas. Dosimetry readers, ensure accurate radiation exposure monitoring. Manual and automated systems for whole body, extremity, neutron, and environmental monitoring are easy to operate, service, and maintain. Extremity dosimeter is a disk dosimeter designed for nuclear power or nuclear medicine personnel that have a high risk of exposure to ionizing radiation, particularly on their hands, due to their work in close proximity to radiation materials and radiation producing equipment.
\end{abstract}

Keywords: Electronic, Extremity, Radiation Dosimeter, New Trend

\section{Introduction}

A radiation dosimeter is a device, instrument or system that measures or evaluates, either directly or indirectly, the quantities exposure, kerma, absorbed dose or equivalent dose, or their time derivatives (rates), or related quantities of ionizing radiation. A dosimeter along with its reader is referred to as a dosimetry system. It has two main uses: for human radiation protection and for measurement of dose in both medical and industrial processes. It Get effective dose monitoring when and where you need it. There are a variety of electronic personal dosimeters, extremity dosimeters, and comprehensive dosimetry management systems to monitor your exposure to ionizing radiation in any work environment $[1,2,3]$. To function as a radiation dosimeter, the dosimeter must possess at least one physical property that is a function of the measured dosimetric quantity and that can be used for radiation dosimetry with proper calibration. In order to be useful, radiation dosimeters must exhibit several desirable characteristics. For example, in radiotherapy exact knowledge of both the absorbed dose to water at a specified point and its spatial distribution are of importance, as well as the possibility of deriving the dose to an organ of interest in the patient. In this context, the desirable dosimeter properties will be characterized by accuracy and precision, linearity, dose or dose rate dependence, energy response, directional dependence and spatial resolution. Obviously, not all dosimeters can satisfy all characteristics. The choice of a radiation dosimeter and its reader must therefore be made judiciously, taking into account the requirements of the measurement situation; for example, in radiotherapy ionization chambers are recommended for beam calibrations [4]. Whilst Dosimetry in its original sense is the measurement of the absorbed dose delivered by ionizing radiation, the term is better known as a scientific sub-specialty in the fields of health physics and medical physics, where it is the 
calculation and assessment of the radiation dose received by the human body. However, Internal dosimetry due to the ingestion or inhalation of radioactive materials relies on a variety of physiological or imaging techniques. External dosimetry, due to irradiation from an external source is based on measurements with a dosimeter, or inferred from other radiological protection instruments. On the other hand, Dosimetry is used extensively for radiation protection and is routinely applied to occupational radiation workers, where irradiation is expected, but regulatory levels must not be exceeded. It is also used where radiation is unexpected, such as in the aftermath of the Three Mile Island, Chernobyl or Fukushima radiological release incidents, where the public irradiation is measured and calculated from a variety of indicators such as ambient measurements of radiation and radioactive contamination. Other significant areas are medical dosimetry, where the required treatment absorbed dose and any collateral absorbed dose is monitored, and in environmental dosimetry, such as radon monitoring in buildings [4].

\section{Methods}

The study comprises a personal selection of recent reports from radiology journals and Medline searches which highlighted the new trend in radiation dosimeters.

\section{Results and Discussion}

\subsection{Radiation Dosimeters and Dosimetry Systems [4]}

It comes in many shapes and forms, and they rely on numerous physical effects for storage and readout of the dosimetric signal. The four most commonly used radiation dosimeters are:

\subsubsection{Ionization Chamber Dosimetry Systems}

Ionization chambers are used in radiotherapy and in diagnostic radiology for the determination of radiation dose. The dose determination in reference irradiation conditions is also called beam calibration. Ionization chambers come in various shapes and sizes, depending upon the specific requirements, but generally they all have the following properties: a) An ionization chamber is basically a gas filled cavity surrounded by a conductive outer wall and having a central collecting electrode. The wall and the collecting electrode are separated with a high-quality insulator to reduce the leakage current when a polarizing voltage is applied to the chamber. b) A guard electrode is usually provided in the chamber to further reduce chamber leakage. The guard electrode intercepts the leakage current and allows it to flow to ground, bypassing the collecting electrode. It also ensures improved field uniformity in the active or sensitive volume of the chamber, with resulting advantages in charge collection. c) Measurements with open air ionization chambers require temperature and pressure correction to account for the change in the mass of air in the chamber volume, which changes with the ambient temperature and pressure. The ionization chambers dosimeter can be: a) Chambers and electrometers. b) Cylindrical (thimble type) ionization chambers. c) Parallel-plate (plane-parallel) ionization chambers. d) Brachytherapy chambers. e) Extrapolation chambers.

\subsubsection{Film Dosimetry}

\section{(i) Radiographic Films}

Radiographic X ray film performs several important functions in diagnostic radiology, radiotherapy and radiation protection. It can serve as a radiation detector, a relative dosimeter, a display device and an archival medium. Unexposed X ray film consists of a base of thin plastic with a radiation sensitive emulsion (silver bromide $(\mathrm{AgBr})$ grains suspended in gelatin) coated uniformly on one or both sides of the base. Ionization of $\mathrm{AgBr}$ grains, as a result of radiation interaction, forms a latent image in the film. This image only becomes visible (film blackening) and permanent subsequently to processing. Light transmission is a function of the film opacity and can be measured in terms of optical density (OD) with devices called densitometers. The OD is defined as OD $=\log 10$ (I0/I) and is a function of dose. $\mathrm{I} 0$ is the initial light intensity and $\mathrm{I}$ is the intensity transmitted through the film. Film gives excellent 2-D spatial resolution and, in a single exposure, provides information about the spatial distribution of radiation in the area of interest or the attenuation of radiation by intervening objects. The useful dose range of film is limited and the energy dependence is pronounced for lower energy photons. The response of the film depends on several parameters, which are difficult to control. Consistent processing of the film is a particular challenge in this regard. Typically, film is used for qualitative dosimetry, but with proper calibration, careful use and analysis film can also be used for dose evaluation. Various types of film are available for radiotherapy work (e.g. direct exposure non-screen films for field size verification, phosphor screen films used with simulators and metallic screen films used in portal imaging). Unexposed film would exhibit a background OD called the fog density (ODf). The density due to radiation exposure, called the net OD, can be obtained from the measured density by subtracting the fog density. OD readers include film densitometers, laser densitometers and automatic film scanners.

\section{(ii) Radiochromic Film}

Radiochromic film is a new type of film in radiotherapy dosimetry. The most commonly used is a GafChromic film. It is a colorless film with a nearly tissue equivalent composition $(9.0 \%$ hydrogen, $60.6 \%$ carbon, $11.2 \%$ nitrogen and $19.2 \%$ oxygen) that develops a blue color upon radiation exposure. Radiochromic film contains a special dye that is polymerized upon exposure to radiation. The polymer absorbs light, and the transmission of light through the film can be measured with a suitable densitometer. Radiochromic film is self-developing, requiring neither developer nor fixer. Since radiochromic film is grainless, it has a very high resolution and can be used in high dose gradient regions for 
dosimetry (e.g. measurements of dose distributions in stereotactic fields and in the vicinity of brachytherapy sources). Dosimetry with radiochromic films has a few advantages over radiographic films, such as ease of use; elimination of the need for darkroom facilities, film cassettes or film processing; dose rate independence; better energy characteristics (except for low energy $\mathrm{X}$ rays of $25 \mathrm{kV}$ or less); and insensitivity to ambient conditions (although excessive humidity should be avoided). Radiochromic films are generally less sensitive than radiographic films and are useful at higher doses, although the dose response nonlinearity should be corrected for in the upper dose region. Radiochromic film is a relative dosimeter. If proper care is taken with calibration and the environmental conditions, a precision better than $3 \%$ is achievable. Data on the various characteristics of radiochromic films (e.g. sensitivity, linearity, uniformity, reproducibility and post-irradiation stability) are available in the literature.

\subsubsection{Luminescence Dosimetry}

Some materials, upon absorption of radiation, retain part of the absorbed energy in metastable states. When this energy is subsequently released in the form of ultraviolet, visible or infrared light, the phenomenon is called luminescence. Two types of luminescence, fluorescence and phosphorescence, are known, which depend on the time delay between stimulation and the emission of light. Fluorescence occurs with a time delay of between $10-10$ and $10-8$ s; phosphorescence occurs with a time delay exceeding 10-8 s. The process of phosphorescence can be accelerated with a suitable excitation in the form of heat or light. If the exciting agent is heat, the phenomenon is known as thermoluminescence and the material is called a thermoluminescent material, or a TLD when used for purposes of dosimetry. If the exciting agent is light, the phenomenon is referred to as optically stimulated luminescence (OSL). The highly energetic secondary charged particles, usually electrons, which are produced in the primary interactions of photons with matter, are mainly responsible for the photon energy deposition in matter. In a crystalline solid these secondary charged particles release numerous low energy free electrons and holes through ionizations of atoms and ions. The free electrons and holes thus produced will either recombine or become trapped in an electron or hole trap, respectively, somewhere in the crystal. The traps can be intrinsic or can be introduced in the crystal in the form of lattice imperfections consisting of vacancies or impurities. Two types of trap are known in general: storage traps and recombination centres. 1) A storage trap merely traps free charge carriers and releases them during the subsequent (a) heating, resulting in the thermoluminescence process, or (b) irradiation with light, resulting in the OSL process. 2) A charge carrier released from a storage trap may recombine with a trapped charge carrier of opposite sign in a recombination centre (luminescence centre). The recombination energy is at least partially emitted in the form of ultraviolet, visible or infrared light that can be measured with photodiodes or photomultiplier tubes (PMTs). The types of Luminescence Dosimetry were:

1. Thermoluminescence

2. Thermoluminescent dosimeter systems

3. Optically stimulated luminescence systems

\subsubsection{Semiconductor Dosimetry}

\section{(i) Silicon Diode Dosimetry Systems}

A silicon diode dosimeter is a $\mathrm{p}-\mathrm{n}$ junction diode. The diodes are produced by taking $\mathrm{n}$ type or $\mathrm{p}$ type silicon and counter-doping the surface to produce the opposite type material. These diodes are referred to as $\mathrm{n}-\mathrm{Si}$ or $\mathrm{p}-\mathrm{Si}$ dosimeters, depending upon the base material. Both types of diode are commercially available, but only the $\mathrm{p}-\mathrm{Si}$ type is suitable for radiotherapy dosimetry, since it is less affected by radiation damage and has a much smaller dark current. Radiation produces electron-hole $(\mathrm{e}-\mathrm{h})$ pairs in the body of the dosimeter, including the depletion layer. The charges (minority charge carriers) produced in the body of the dosimeter, within the diffusion length, diffuse into the depleted region. They are swept across the depletion region under the action of the electric field due to the intrinsic potential. In this way a current is generated in the reverse direction in the diode.

Diodes are used in the short circuit mode, since this mode exhibits a linear relationship between the measured charge and dose. They are usually operated without an external bias to reduce leakage current. Diodes are more sensitive and smaller in size than typical ionization chambers. They are relative dosimeters and should not be used for beam calibration, since their sensitivity changes with repeated use due to radiation damage. Diodes are particularly useful for measurement in phantoms, for example of small fields used in stereotactic radiosurgery or high dose gradient areas such as the penumbra region. They are also often used for measurements of depth doses in electron beams. For use with beam scanning devices in water phantoms, they are packaged in a waterproof encapsulation. When used in electron beam depth dose measurements, diodes measure directly the dose distribution (in contrast to the ionization measured by ionization chambers). Diodes are widely used in routine in vivo dosimetry on patients or for bladder or rectum dose measurements. Diodes for in vivo dosimetry are provided with buildup encapsulation and hence must be appropriately chosen, depending on the type and quality of the clinical beams. The encapsulation also protects the fragile diode from physical damage. Diodes need to be calibrated when they are used for in vivo dosimetry, and several correction factors have to be applied for dose calculation. The sensitivity of diodes depends on their radiation history, and hence the calibration has to be repeated periodically. Diodes show a variation in dose response with temperature (this is particularly important for long radiotherapy treatments), dependence of signal on the dose rate (care should be taken for different source to skin distances), angular (directional) dependence and energy dependence even for small variations in the spectral composition of radiation beams (important for 
the measurement of entrance and exit doses).

\section{(ii) MOSFET Dosimetry Systems}

A metal-oxide semiconductor field effect transistor (MOSFET), a miniature silicon transistor, possesses excellent spatial resolution and offers very little attenuation of the beam due to its small size, which is particularly useful for in vivo dosimetry. MOSFET dosimeters are based on the measurement of the threshold voltage, which is a linear function of absorbed dose. Ionizing radiation penetrating the oxide generates charge that is permanently trapped, thus causing a change in threshold voltage. The integrated dose may be measured during or after irradiation. MOSFETs require a connection to a bias voltage during irradiation. They have a limited lifespan.

A single MOSFET dosimeter can cover the full energy range of photons and electrons, although the energy response should be examined, since it varies with radiation quality. For megavoltage beams, however, MOSFETs do not require energy correction, and a single calibration factor can be used. MOSFETs exhibit small axial anisotropy $\left( \pm 2 \%\right.$ for $\left.360^{\circ}\right)$ and do not require dose rate corrections. Similarly, to diodes, single MOSFETs exhibit temperature dependence, but this effect has been overcome by specially designed double detector MOSFET systems. In general, they show nonlinearity of response with the total absorbed dose; however, during their specified lifespan, MOSFETs retain adequate linearity. MOSFETs are also sensitive to changes in the bias voltage during irradiation (it must be stable), and their response drifts slightly after the irradiation (the reading must be taken in a specified time after exposure). MOSFETs have been in use for the past few years in a variety of radiotherapy applications for in vivo and phantom dose measurements, including routine patient dose verification, brachytherapy, TBI, intensity modulated radiotherapy (IMRT), intraoperative radiotherapy and radiosurgery. They are used with or without additional buildup, depending on the application.

The strengths and weaknesses of these four dosimeters are summarized in Table 1.

Table 1. Main Advantages and disadvantages of The Four Commonly Used Dosimetric Systems.

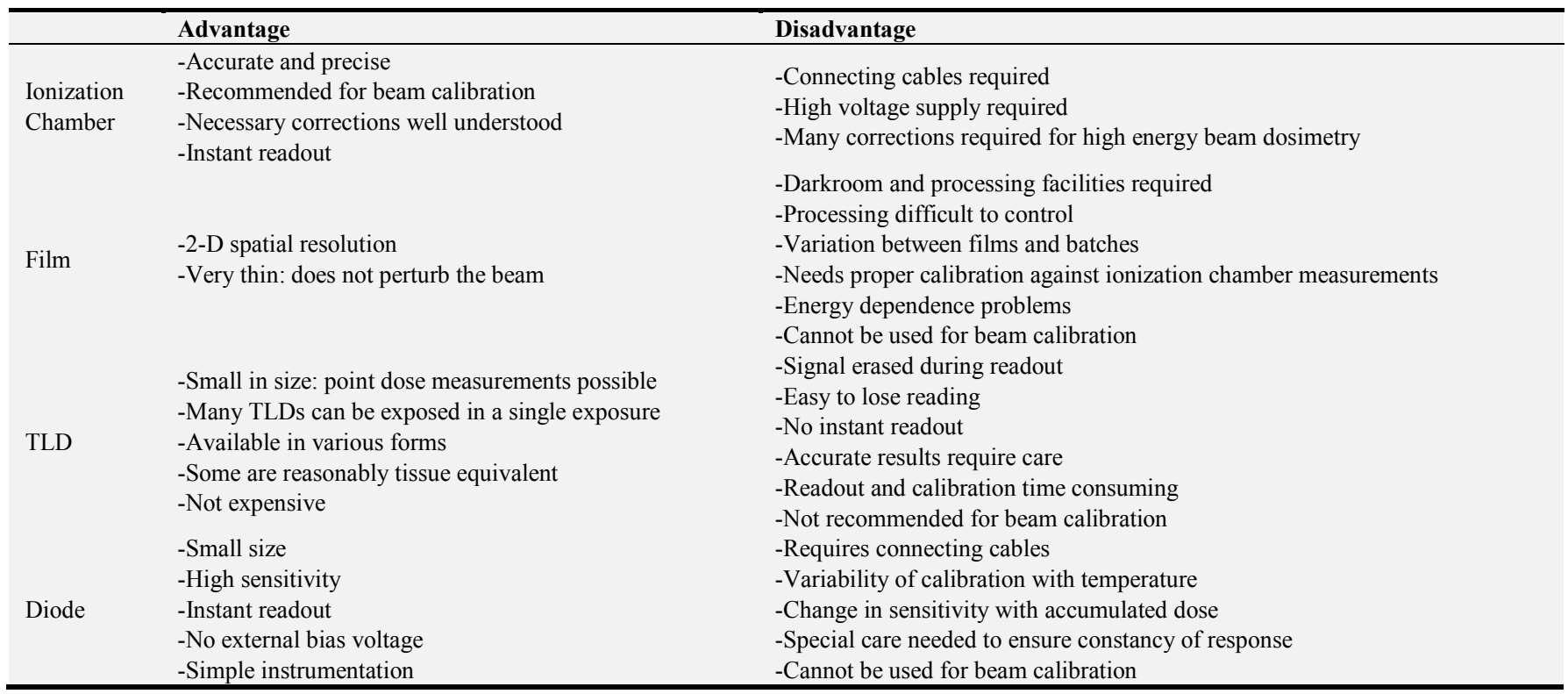

\subsection{Other Dosimetry Systems}

1. Alanine/electron paramagnetic resonance dosimetry system

2. Plastic scintillator dosimetry system

3. Diamond dosimeters

4. Gel dosimetry systems

\subsection{Personal Dosimeters [5]}

The personal ionizing radiation dosimeter is of fundamental importance in the disciplines of radiation dosimetry and radiation health physics and is primarily used to estimate the radiation dose deposited in an individual wearing the device. Ionizing radiation damage to the human body is cumulative, and is related to the total dose received, for which the SI unit is the Sievert. Workers exposed to radiation, such as radiographers, nuclear power plant workers, doctors using radiotherapy, those in laboratories using radionuclides, and HAZMAT teams (personnel specially trained to handle dangerous goods, which include materials that are radioactive, flammable, explosive, corrosive, oxidizing, asphyxiating, biohazardous, toxic, pathogenic, or allergenic.) are required to wear dosimeters so a record of occupational exposure can be made. Such devices are known as "legal dosimeters" if they have been approved for use in recording personnel dose for regulatory purposes. Dosimeters can be worn to obtain a whole body dose and there are also specialist types that can be worn on the fingers or clipped to headgear, to measure the localized body irradiation for 
specific activities.

The common types of personal dosimeters for ionizing radiation include:

\section{(i) Electronic Personal Dosimeter (EPD)}

The electronic personal dosimeter (EPD) is an electronic device that has a number of sophisticated functions, such as continual monitoring which allows alarm warnings at preset levels and live readout of dose accumulated. These are especially useful in high dose areas where residence time of the wearer is limited due to dose constraints. The dosimeter can be reset, usually after taking a reading for record purposes, and thereby re-used multiple times.

\section{(ii) MOSFET Dosimeter}

MOSFET dosimeters are now used as clinical dosimeters for radiotherapy radiation beams. The main advantages of MOSFET devices are:

1. The MOSFET dosimeter is direct reading with a very thin active area (less than $2 \mu \mathrm{m}$ ).

2. The physical size of the MOSFET when packaged is less than $4 \mathrm{~mm}$.

3. The post radiation signal is permanently stored and is dose rate independent. [6]

Gate oxide of MOSFET which is conventionally silicon dioxide is an active sensing material in MOSFET dosimeters. Radiation creates defects (acts like electron-hole pairs) in oxide, which in turn affects the threshold voltage of the MOSFET. This change in threshold voltage is proportional to radiation dose. Alternate high-k gate dielectrics like Hafnium dioxide [7] and Aluminum oxides are also proposed as radiation dosimeters.

\section{(iii) Film Badge Dosimeter}

Film badge dosimeters are for one-time use only. The level of radiation absorption is indicated by a change to the film emulsion, which is shown when the film is developed.

(iv) Quartz Fiber Dosimeter

Quartz fiber dosimeters are charged to a high voltage. As the gas in the dosimeter chamber becomes ionized by radiation the charge leaks away, causing the fiber indicator to change against a graduated scale [8].

(v) Thermoluminescent Dosimeter (TLD)

A thermoluminescent dosimeter measures ionizing radiation exposure by measuring the intensity of visible light emitted from a crystal in the detector when heated. The intensity of light emitted is dependent upon the radiation exposure.

Both the quartz fiber and film badge types are being superseded by the TLD and the EPD.

\subsection{Thermo Scientific DXT-RAD Extremity Dosimeter [9]}

Radiation workers who work closely with radioactive materials and radiation producing equipment are particularly susceptible to over-exposure on their hands. This requires monitoring skin radiation dose on the fingers or wrists, exactly where the exposure is likely to be the highest. Detect and measure photon or beta radiation dose closest to the source using the Thermo Scientific ${ }^{\mathrm{TM}}$ DXT-RAD Extremity Dosimeter (figure 1). It provides Radiation Safety Managers the ability to determine their personnel's highest potential exposure to drive appropriate corrective action. The small and versatile single-element dosimeters fit comfortably into adjustable rings and wrist bands, ensuring wearability without interfering with the task at hand. It utilizes the same high-quality and reliable thermoluminescent dosimetry material of the Thermo Scientific Harshaw TLD multielement card dosimeters and offers additional advantages over other market options including, permanent individual barcodes, hot or cold sterilization, fast read out and near tissue equivalency. These features, combined with compatibility with the broader Thermo Scientific Harshaw TLD system, including high capacity readers and software programs makes the DXT-RAD the ideal solution for extremity dosimetry. The Thermo Scientific DXT-RAD Extremity dosimeter is a disk dosimeter designed for nuclear power or nuclear medicine personnel that have a high risk of exposure to ionizing radiation, particularly on their hands, due to their work in close proximity to radiation materials and radiation producing equipment. The Key Features and Benefits are:

1. Permanent Individual Barcodes - Ensure unbroken chain of custody

2. Hot or Cold Sterilization - Enjoy flexibility in processing

3. Fast Readout - Recognize efficiency in processing by reading out the DXT-RAD in less than 30 seconds

4. TLD Material to meet application needs - Select from a range of TLD materials and filtration for use in specific radiation environments

5. System Compatibility - Process DXT-RAD Dosimeters in the same Thermo Scientific Harshaw TLD Readers as traditional TLD multi-element card dosimeters, utilizing DXT-RAD Carrier Cards with capacity for four extremity dosimeters.

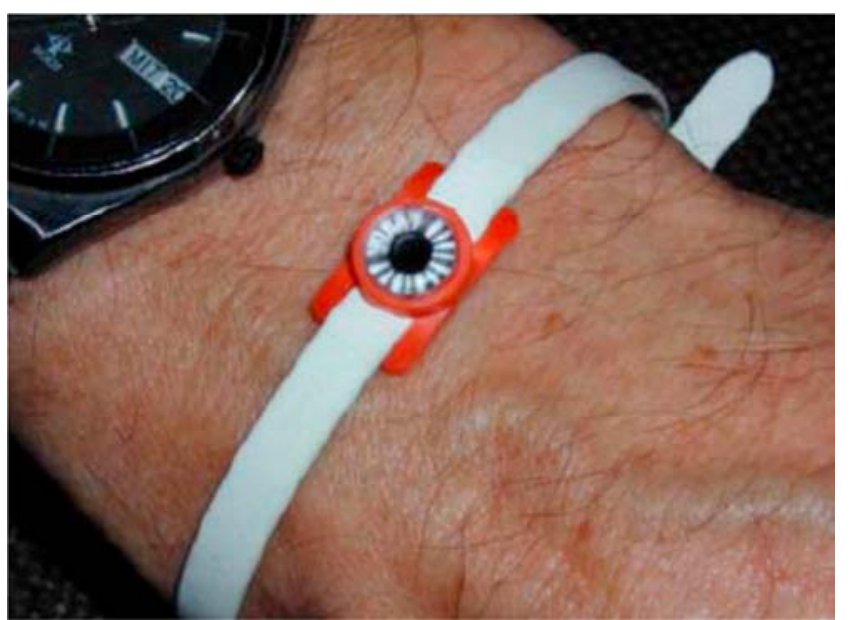

Figure 1. Thermo Scientific ${ }^{T M} D X T-R A D$ Extremity Dosimeter. 


\subsection{Area Monitoring Dosimeter}

\subsubsection{RadEye Product Family [10]}

With the RadEye Product Family, Thermo Fisher Scientific offers a wide range of advanced handheld instruments for radiation detection, gamma dose rate measurements and area monitoring (figure 2).

\section{(i) Rad Eye - The Next Generation of Radiation Meters [10]}

Thermo Scientific offers a comprehensive range of advanced instruments for radiation detection, dose rate and contamination measurements. The characteristic features of this versatile pocket meter are the small size, the ease and flexibility of operation and its superior measurement performance, which is provided by the use of sophisticated low power technology. Fully automated self-diagnosis minimizes required maintenance. All essential functions are easily accessed, even while wearing protective gloves. The top-mounted alarm-LED can be seen while the instrument is worn in a belt-holster. A built-in vibrator and an earphoneoutput provide silent alarming for use in very noisy environments.

\section{(ii) Features}

a) Rugged and reliable.

b) Large graphic display.

c) Lightweight instruments, starting from $160 \mathrm{~g}$.

d) Simple and intuitive user interface.

e) Easily configured for specific tasks.

f) Durable and shock resist.

g) Accurate with excellent EMI immunity.

h) Low power technology.

i) Use of rechargeable standard-size batteries.

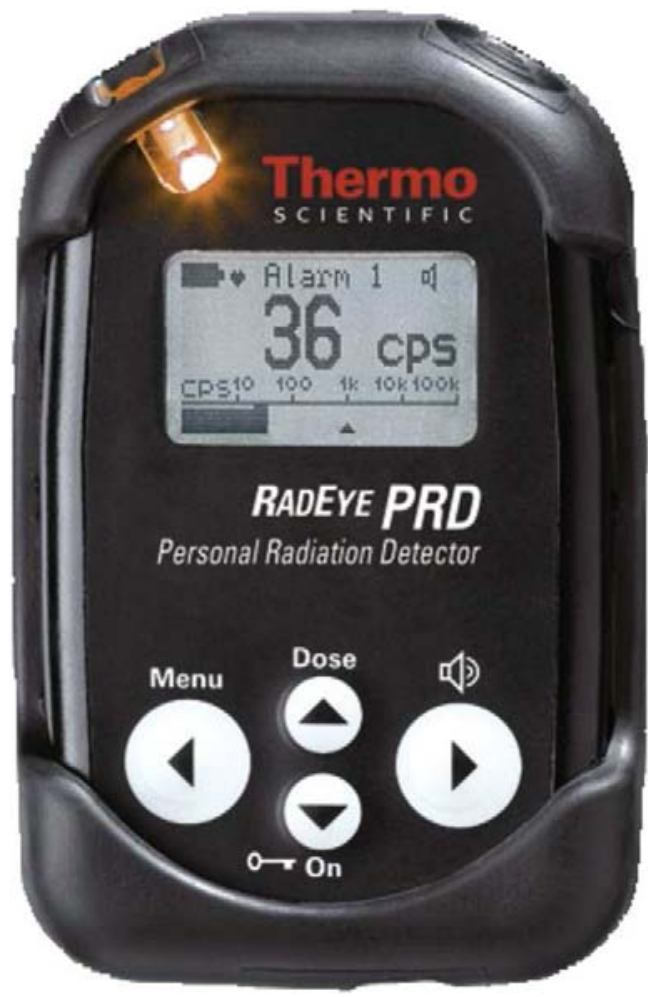

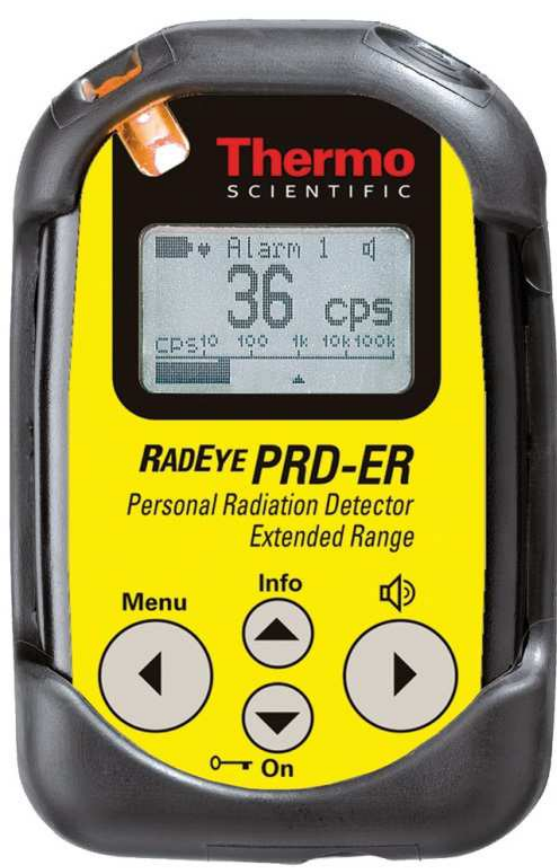

Figure 2. Rad Eye Product Family.

\subsubsection{Radiation Protection Dose Quantities}

External radiation dose quantities used in radiological protection - based on ICRU report 57. The dosimeter plays an important role within the international radiation protection system developed by the International Commission on Radiological Protection (ICRP) and the International Commission on Radiation Units and Measurements (ICRU).

\section{(i) Protection Quantities}

The protection quantities are used as "limiting quantities" to specify exposure limits to ensure that the occurrence of stochastic health effects is kept below unacceptable levels and that tissue reactions are avoided $[11,12,13]$. These quantities cannot be practically measured and are a calculated value of irradiation of organs of the human body, which is arrived at by using an anthropomorphic phantom. This is a 3D computational model of the human body which attempts to take into account a number of complex effects such as body self-shielding and internal scattering of radiation. As protection quantities cannot practically be measured, operational quantities are used to relate them to practical radiation instrument and dosimeter responses. [12, 13]

\section{(ii) Operational Quantities}

Operational quantities are aimed at providing an estimate or upper limit for the value of the protection quantities related to an exposure. They are used in practical regulations or guidance. These relate real-life operational instrument measurements and responses to the calculated protection quantities. [12] Personal dose equivalent, $\mathrm{Hp} \mathrm{(d),} \mathrm{is} \mathrm{defined}$ by the ICRP as the dose equivalent in soft tissue at an appropriate depth, $\mathrm{d}$, below a specified point on the human body. The specified point is usually given by the position where the individual's dosimeter is worn. $[12,13]$ 


\section{(iii) Instrument and Dosimeter Response}

This is an actual reading obtained from such as an ambient dose gamma monitor, or a personal dosimeter. The dosimeter is calibrated in a known radiation field to ensure display of accurate operational quantities and allow a relationship to known health effect. The personal dose equivalent is used to assess dose uptake, and allow regulatory limits to be met. It is the figure usually entered into the records of external dose for occupational radiation workers.

\section{(iv) Dosimeter Calibration}

The "slab" phantom is used to represent the human torso for calibration of whole body dosimeters. The IAEA states "The slab phantom is $300 \mathrm{~mm} \times 300 \mathrm{~mm} \times 150 \mathrm{~mm}$ depth to represent the human torso". [14]

\section{(v) Process Irradiation Verification}

Manufacturing processes that treat products with ionizing radiation, such as food irradiation, use dosimeters to calibrate doses deposited in the matter being irradiated. These usually must have a greater dose range than personal dosimeters, and doses are normally measured in the unit of absorbed dose: the gray (Gy). The dosimeter is located on or adjacent to the items being irradiated during the process as a validation of dose levels received (figure 3 ).

Gallery

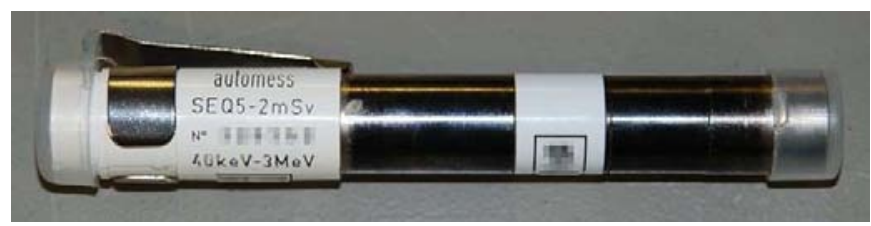

QFD - These are now largely superseded radiometer-dosimeter (Front view).

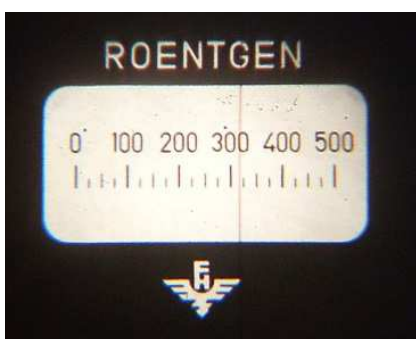

View of QFD reading.

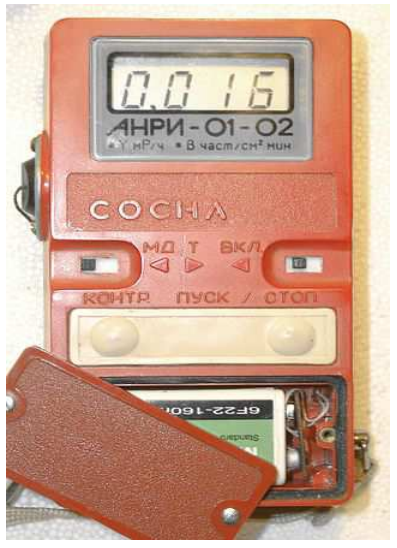

Russian Sosna.

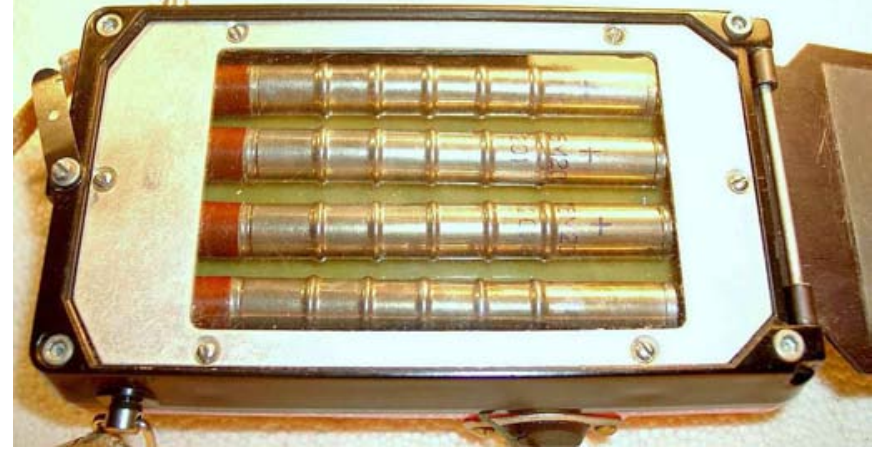

Russian Sosna radiometer-dosimeter (Rear view) Soeks 01M.
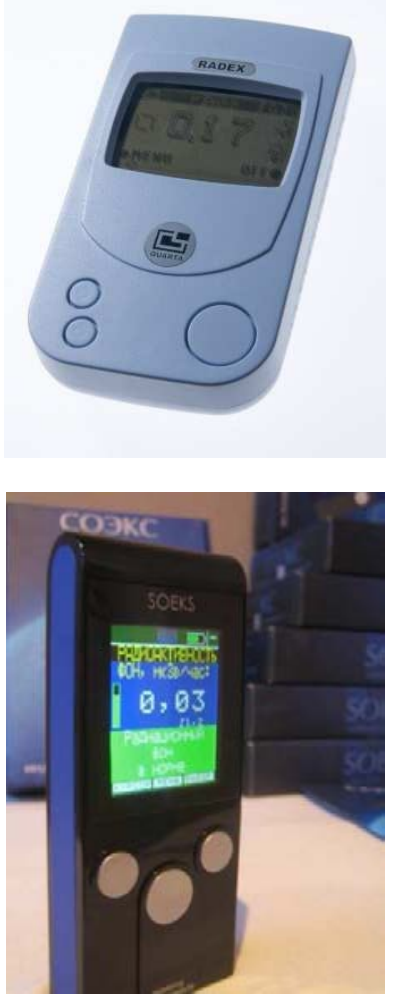

Russian Radex RD1503

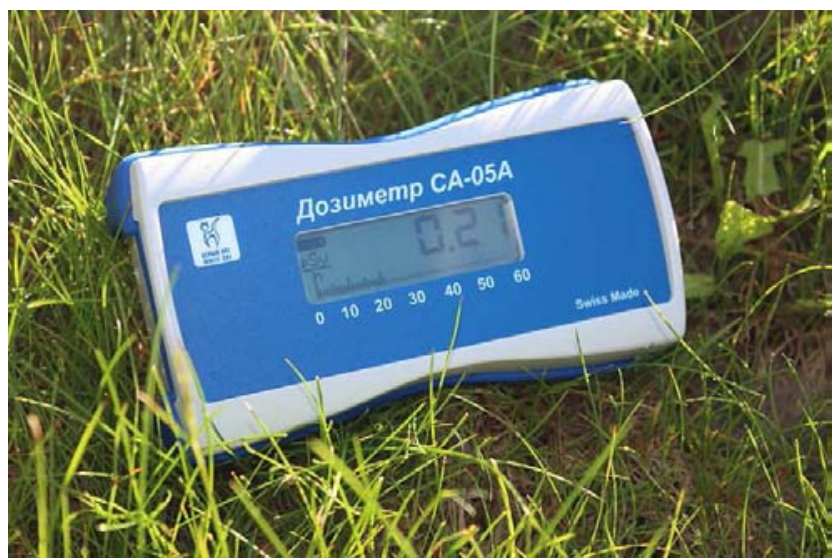

Swiss dosimeter SA-05A.

Figure 3. Manufacturing processes that treat products with ionizing radiation. 


\subsubsection{Electronic Radiation Dosimeter}

Electronic dosimeters protect people from radiation and limit access to dangerous areas. It protects the wearer from the harmful effects of radiation by tracking changes in exposure and keeping an ongoing record of the user's dose over time. Combined with access control systems, it is possible to limit total exposure to radiation and control access to radiological areas.

\section{(i) DMC 3000 Electronic Dosimeter}

The DMC 3000 Electronic Dosimeter is a great fit for almost any application where a live indication of both total radiation dose and dose rate is needed. It protects workers who are exposed to radiation by notifying them of changing levels of radiation exposure with ultra-bright LEDs, audible alarms and vibration (figure 4). [14]
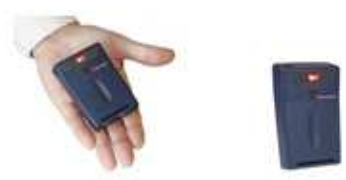

A

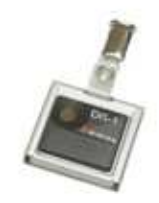

F
B
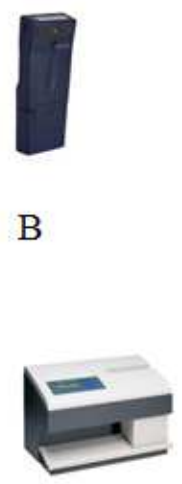

G

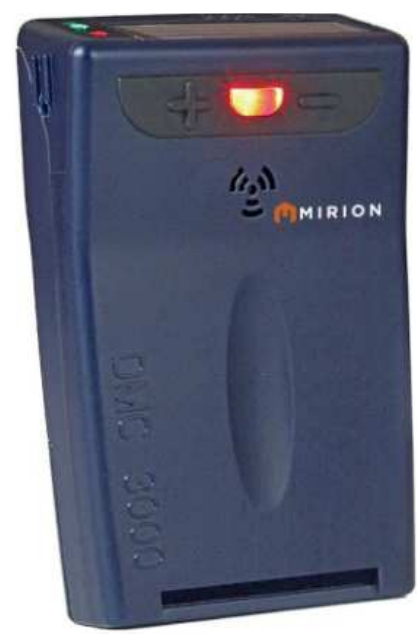

Figure 4. DMC 3000 Electronic Radiation Dosimeter.

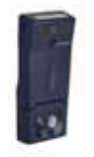

$\mathrm{C}$

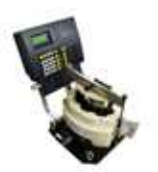

$\mathrm{H}$

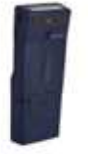

$\mathrm{D}$

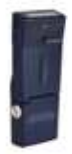

$\mathrm{E}$

Figure 5. A: DMC 3000 Electronic Radiation Dosimeter, B: DMC 3000 PRD Module, C: DMC 3000 Beta Module, D: DMC 3000 Neutron Module, E: DMC 3000 Telemetry Module, F: DIS Dosimetry System, G: TLD Dosimetry System, H: Dosimeter Calibrators, I: Access Control Hardware.

\section{(a) DMC 3000 Modules}

The DMC 3000 Personal Electronic Radiation Dosimeter [14] represents 25 years of refinement in electronic dosimetry. It features superior gamma and X-ray energy response, programmable alarms with visual LED, audible, and vibrating alarm indicators, simple 2-button navigation, and the ability to be fitted with external modules for expanded capabilities.

The DMC 3000 has a complete line of attachable modules that expand the detection and communications capabilities of the dosimeter:

1. The DMC 3000 Beta Module.

2. The DMC 3000 Neutron Module.

3. The DMC 3000 Telemetry Module.

\section{(b) Product Features [14]}

a. Designed for ruggedness and durability.

b. Loud audible alarms, coupled with ultrabright LEDs and vibration capability.

c. Simple 2-button operation and navigation of display options.

d. Meets or exceeds applicable IEC and ANSI standards. e. Operates for up to 9 months on a single standard AAA battery.

\section{(c) Appropriate for [14]}

a. Nuclear Power

b. Industry and Manufacturing

c. Laboratories and Education

d. Military \& Homeland Security

e. Healthcare

\section{(ii) DIS Dosimetry System}

Combining reliable ionization chamber technology with an electronic Direct Ion Storage memory cell, the DIS Series of personal electronic radiation dosimeters offers several features not available on most passive dosimeters. They can be quickly and repeatedly read on-site, and have the capability to operate in pulsed fields. Dosimeter can be reset to display doses for either specific time periods (day, week, month) or specific tasks. Dose information is recorded automatically into a database every time a read-out is performed. Personnel doses are stored for legal reporting (background radiation is subtracted automatically). It can measure in pulsed field environments [14]. 


\subsection{Radiation Monitoring Services}

\subsubsection{Pocket Dosimeter}

(i) Instadose+ $[15,16]$

1. Pushing the boundaries of dosimetry technology (figure 6) $[15,16]$

2. Instadose + dosimeters allow for improved dosimetry compliance, while simplifying dose reads via iPhone, iPad, or PC at any time. With Instadose+, you can closely track exposure and pursue lower dose thresholds for high risk employees.

3. The instadose+ dosimetry system revolutionizes the way RSOs manage their radiation monitoring programs. From automatic dose reads captured by iPhones, iPads, or PCs; to high dose notification alerts and online badge reassignments - this system simplifies the administration of any radiation monitoring program.

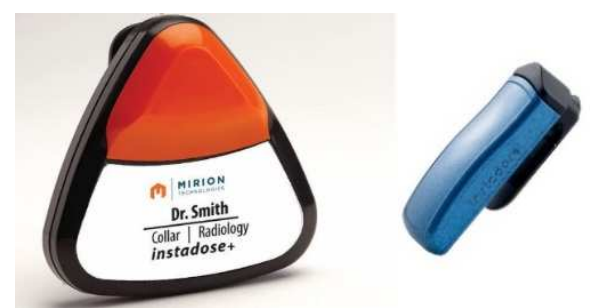

Figure 6. Instadose+ and instadose 1.

\section{(ii) Instadose 1 Dosimeter (x-ray Badge)}

Immediate dose readings can be performed from any computer with internet access by connecting the instadose dosimeter (x-ray badge) to your USB port and logging into your secure online account. Based upon proprietary direct ion storage technology, the instadose device includes access to each users historical and current exposure readings online. There is no need to send badges to a processing center, simplifying administration and reducing costs $[15,16]$.

\section{(a) Dosimetry Made Easy by: [15, 16]}

1. Elimination of the badge collection process

2. Increased compliance

3. Instant dose reads captured with iPhones ${ }^{\circledR}$, iPads ${ }^{\circledR}$, PCs, or instaLink stations

4. Online Report access eliminates the need to archive and distribute results

5. RSO-defined read intervals for dose trending

6. The ability to track and lower dose for high risk employees

7. Immediate e-mail notification when wearer exceeds dose level specified

\section{(b) Product Description: $[15,16]$}

Immediate dose readings can be performed from any computer with internet access by connecting the instadose dosimeter (x-ray badge) to your USB port and logging into your secure online account. Based upon proprietary direct ion storage technology, the instadose device includes access to each user's historical and current exposure readings online.
There is no need to send badges to a processing center, simplifying administration and reducing costs.

(c) Benefits for Your Facility Include: [15, 16]

a) Accredited by NVLAP (Lab Code 100555-0), HSE, FANR-R, Nigerian Nuclear Regulatory Authority (NNRA), and Australia States New South Wales and Victoria

b) Unlimited real-time dose readings online

c) Online badge reassignment

d) Dose reports maintained online

e) NO badge collection

f) NO battery replacements or calibration

\subsubsection{The Genesis Ultra TLD Dosimeter}

The Genesis TLD Dosimeter line offers improved performance and superior user factors. This dosimeter responds accurately to beta, gamma, X-ray and neutron radiation. The response of each element is adjusted by the application of its own unique correction factor. These dosimeters report deep dose, lens of eye and shallow doses. Unlike other TLD products, the Genesis Ultra TLD has virtually no fading characteristics. Due to the increase in signal response a minimum reportable dose (MRD) as low as $1 \mathrm{mrem}(0.01 \mathrm{mSv})$ is available, compared to the 10 mrem $(0.10 \mathrm{mSv}) \mathrm{MRD}$ of other TLD products (figure 7$)$ [17].

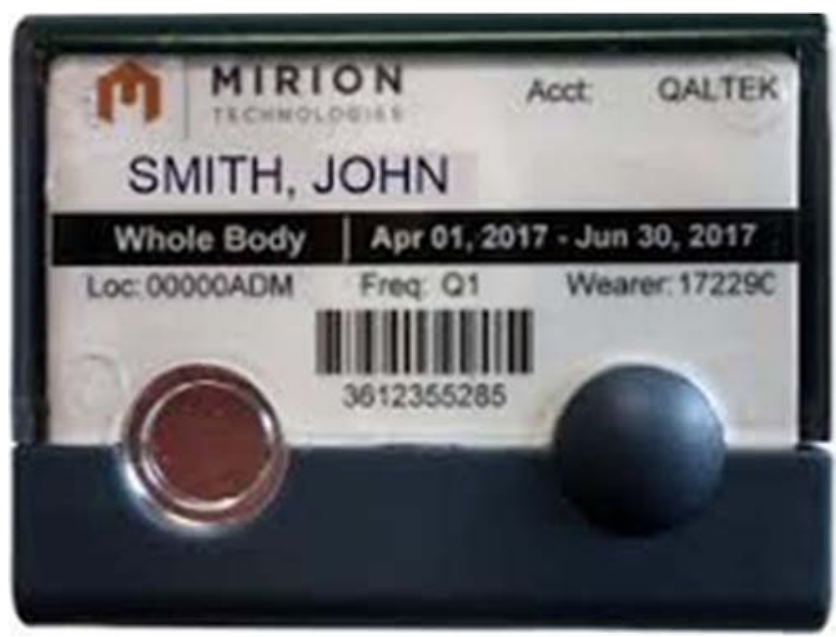

Figure 7. The Genesis Ultra Dosimeters.

\subsubsection{The APex Dosimeters}

The APex dosimeters slim profile and light weight (2.8" $x$ 1.6") makes the badge easy to wear on the body or extremities. Even when exposed to extreme temperatures or moisture the dosimeter will provide accurate dose readings. As with other OSL technologies, APex can be re-read multiple times, enabling re-evaluation into unusual exposures. Wear periods range from one week to one year; you decide what works best for your radiation monitoring program. It can be used by medical practitioners and technologists, dentist, veterinarians, chiropractors, or any practice with potential exposure to photon radiation (figure 8) [18]. 

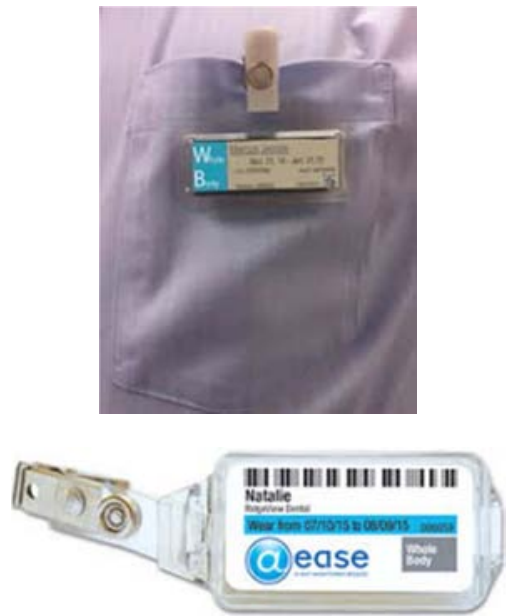

Figure 8. The Genesis Ultra and The APex TLD Dosimeters.

\section{Conclusion}

A radiation dosimeter is a device that measures exposure to ionizing radiation. It is used for human radiation protection as a measurement of dose in both medical and industrial processes. This study aimed to identify and highlight the new technology in the types of radiation dosimeters. Radiation dosimeters and dosimetry systems come in many shapes and forms. They rely on numerous physical effects for storage and readout of the dosimetric signal. There are a variety of electronic dosimetry systems that can monitor any work environment. Manual and automated systems for whole body, extremity, neutron, and environmental monitoring are easy to operate, service, and maintain.

\section{References}

[1] Seco, J., Clasie, B. \& Partridge, M. (2014). Review on the characteristics of radiation detectors for dosimetry and imaging. Phys. Med. Biol. 59 (20), R303-R347. doi:10.1088/0031-9155/59/20/R303.

[2] Hill, R., Healy, B., Holloway, L., Kuncic, Z., Thwaites, D. \& Baldock, C. (2014). Advances in kilovoltage x-ray beam dosimetry. Phys. Med. Biol. 59 (6), R183-R231. doi:10.1088/0031-9155/59/6/R183.

[3] Baldock, C., Deene, Y. De., Doran, S., Ibbott, G., Jirasek, A., Lepage, M., McAuley, K. B., Oldham, M. \& Schreiner, L. J. (2010). Polymer gel dosimetry. Phys. Med. Biol. 55 (5), R1R63. doi:10.1088/0031-9155/55/5/R01.

[4] Izewska, J. \& Rajan, G. (2012). Radiation dosimeters. Chapter 3. Dosimetry and Medical Radiation Physics. Pdf Access: nov. 11. Available: http://www.naweb.iaea.org/nahu/dmrp/pdf_files/Chapter3.

[5] Electronic Personal Dosimeter (EPD) (2016). From Wikipedia, the free encyclopedia. Wikipedia ${ }^{\circledR}$ is a registered trademark of the Wikimedia Foundation, Inc., a non-profit organization. Last modified on 29 December 2016, at 18:24. https://en.wikipedia.org/wiki/Dosimeter\#Personal_dosimeters. 5B4.5D.

[6] Electronic Personal Dosimeter (EPD) (2015). From Wikipedia, the free encyclopedia. Wikipedia ${ }^{\circledR}$ is a registered trademark of the Wikimedia Foundation, Inc., a non-profit organization. Last modified on 14 November 2016, at 22:02. https://en.wikipedia.org/wiki/Electronic_Personal_Dosimeter\# MOSFET_dosimeters.

[7] Senthil Srinivasan, V. S. \& Pandya, A. (2011). Dosimetry aspects of hafnium oxide metal-oxide-semiconductor (MOS) capacitor. Thin Solid Films. 520 (1), 574-577. http://dx.doi.org/10.1016/j.tsf.2011.07.010.

[8] Thermo Fisher Scientific (2015), Thermo Scientific DXTRAD, Extremity Dosimeter. (C) 2015 Thermo Fisher Scientific Inc. 150402 DB DXT RAD-e-V1.0. www.thermoscientific.com.

[9] Thermo Fisher Scientific (2012), RadEye Selection Guide, Handheld Detection for Any Scenario, RadEye Product Family. (C2012 Thermo Fisher Scientific Inc. Bluetooth is a trademark of Bluetooth SIG, Inc., Bellevue, Washington, United States. 120809_DB_RadEye_Selection_Guide-e-V3.0. www.thermoscientific.com/rmp.

[10] Paul, F. (2008). Pocket Chambers and Pocket Dosimeters. Health physics historical instrument museum collection. Oak Ridge Associated Universities. Last updated (2007-07-25). Retrieved 2008-11-08.

[11] ICRP. (2007). The 2007 Recommendations of the International Commission on Radiological Protection. ICRP Publication 103. Ann. ICRP. 37 (2-4). ISBN 978-0-70203048-2. Archived from the original on 16 November 2012. Retrieved 17 May 2012.

[12] Dietze, G., Eckerman, K., Menzel, H., Stather, J., Streffer, C., Alberts, W., Balonov, M., Berkovski, V., Bouville, A., Edwards, A., Harrison, J., Lipzstein, J., Pellicioni, M., Phipps, A. \& Pradhan, A. (2005). Basis for dosimetric quantities used in radiological protection. In: International commission on radiological protection, Committee 2, Task Group of ICRP. April 14, 2005, (pp 1-59). Vienna, ICRP 21/110/05.

[13] IAEA Safety report 16 (2000). Calibration of radiation protection monitoring instruments. Safety reports series, ISSN 1020-6450; no. 16, STI/PUB/1074. ISBN 92-0-100100-2.

[14] Mirion Technologies (2014). Radiation. Safety. DMC 3000 Personal Electronic Dosimeter. Copyright (c) 2014 Mirion Technologies, Inc. or its affiliates. www.mirion.com. 1151199EN-G.

[15] Mirion Technologies (2014). instadose+ Dosimeter, Transforming dosimetry with the RSO in mind. Copyright (c) 2014 Mirion Technologies, Inc. or its affiliates. 2652 McGaw, Avenue, Irvine, CA 92614, USA, dsd-support@mirion.com. LIT4394_1_US.

[16] Mirion Technologies (2014). instadose Dosimeter, Instant. Precise, Portable. Copyright (c) 2014 Mirion Technologies, Inc. or its affiliates. Avenue, Irvine, CA 92614, USA, dsdsupport@mirion.com. LIT4386_5v1.

[17] Mirion Technologies (2014). Dosimetry Services Division, Genesis Ultra TLD. Copyright (c) 2014. Mirion Technologies (GDS), Inc. or its affiliates. $2652 \mathrm{McGaw}$ Avenue | Irvine, CA 92614 USA.dsd-support@mirion.com. LIT4373_3.

[18] Mirion Technologies (2014). Dosimetry Services Division, APex Dosimeter. Copyright (c) 2014. Mirion Technologies (GDS), Inc. or its affiliates. $2652 \mathrm{McGaw}$ Avenue | Irvine, CA 92614 USA.dsd-support@mirion.com. LIT4407. 